\title{
DERFIVED
}

\section{in 161997}

$\therefore \mathrm{TI}$ ANL/MSD/CP--9/9/3

THE MECHANISM OF SPUTTER-INDUCED ORIENTATION CHANGE IN YBCO FILMS ON MgO (001)*

$$
\text { CONF- } 961202--58
$$

\author{
Y. Huang, B. V. Vuchic, P. Baldo, and K. L. Merkle \\ Materials Science Division \\ Argonne National Laboratory \\ Argonne, IL 60439 USA \\ D. B. Buchholz, S. Mahajan, J. S. Lei, \\ P. R. Markworth, and R. P. Chang \\ Department of Materials Science and Engineering \\ Northwestern University \\ Evanston, IL 60201 USA
}

December 1996

The submitied manuscript has been created
by the University of Chicago as Operator of
Argonne National Laboratory (Argonne")
under Contract No. W-31-109-ENG-38 with
the U.S. Department of Energy. The U.S.
Government retains for itself, and others
acting on its behalf, a paid-up, non
exclusive, irrevocable worldwide license in
said article to reproduce, prepare derivative
works, distribute copies to the public, and
perform publicly and display publicly, by or
on behalf of the Government.

on behalf of the Governmen

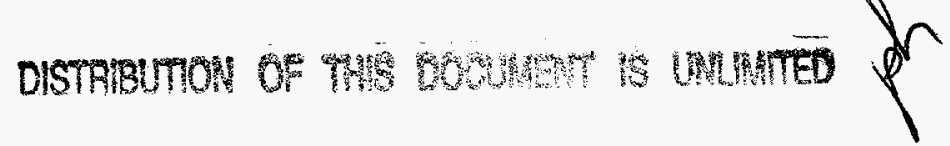

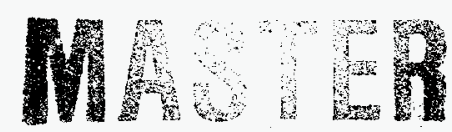

Paper to be presented at the Materials Research Society, December 2-6, 1996, Boston, MA.

\footnotetext{
*Work supported by the U.S. Department of Energy, Basic Energy Sciences-Materials Sciences, under contract \#W-31-109-ENG-38 and the National Science Foundation, under contract \#DMR 91-20000.
} 


\section{DISCLAIMER}

This report was prepared as an account of work sponsored by an agency of the United States Government. Neither the United States Government nor any agency thereof, nor any of their employees, make any warranty, express or implied, or assumes any legal liability or responsibility for the accuracy, completeness, or usefulness of any information, apparatus, product, or process disclosed, or represents that its use would not infringe privately owned rights. Reference herein to any specific commercial product, process, or service by trade name, trademark, manufacturer, or otherwise does not necessarily constitute or imply its endorsement, recommendation, or favoring by the United States Government or any agency thereof. The views and opinions of authors expressed herein do not necessarily state or reflect those of the United States Government or any agency thereof. 


\section{DISCLAIMER}

Portions of this document may be illegible in electronic image products. Images are produced from the best available original document. 
The mechanism of sputter-induced orientation change in YBCO films on $\mathrm{MgO}(001)$

aY.Huang, aB.V.Vuchic, aP. Baldo, bD.B.Buchholz, bS.Mahajan, bJ.S.Lei, bP.R.Markworth, aK.L.Merkle and bR.P.H.Chang

aMaterials Science Division, Argonne National Laboratory

bDept. of Material Science and Engineering, Northwestern University

\section{Abstract}

The mechanisms of the sputter-induced orientation change in $\mathrm{YBa}_{2} \mathrm{Cu}_{3} \mathrm{O}_{7-\mathrm{x}}(\mathrm{YBCO})$ films grown on $\mathrm{MgO}(001)$ substrates by pulsed organometallic beam epitaxy (POMBE) are investigated by X-ray diffraction, Rutherford backscatter spectroscopy (RBS), cross-section TEM (XTEM) and microanalysis. It is found that the $\mathrm{W}$ atom implantation concurring with the ion sputtering plays an important role in effecting the orientation change. This implantation changes the surface structure of the substrate and induces an intermediate layer in the initial growth of the YBCO film, which in turn acts as a template that induces the orientation change. It seems that the surface morphology change caused by ion sputtering has only a minor effect on the orientation change.

\section{Introduction}

The grain boundaries in high-Tc superconductor materials can be used to form Josephson junctions which have important applications in tunable microwave emission, magnetic field measurements and potentially in future electronic devices. Many methods have been developed to fabricate grain boundary junctions (GBJs) in superconductor thin films. One of the most popular methods is bi-epitaxial film growth. The method discussed in this paper is similar to but simpler than the bi-epitaxy method. Instead of using a seed layer the substrates are treated with low energy ion sputtering to change the orientation of the superconductor films $[1,2]$. Under favorable deposition conditions the superconductor films grow with different in-plane rotation on the sputtered and non-sputtered areas and thus form a GBJ between the two areas.

Sputter induced epitaxy modification (SIEM) was first reported by Chew et al[3] in coevaporated YBCO films on $\mathrm{MgO}(001)$ substrates and later observed by Vuchic et al.[1] and Buchholz et al[2] in YBCO films grown by the POMBE method. In all of these cases the YBCO films are c-axis oriented. The in-plane orientation relations reported by Buchholz et al.[2] are $[110]_{\mathrm{YBCO}} / /[100]_{\mathrm{MgO}}$ in the non-sputtered regions of the $\mathrm{MgO}(001)$ substrate and $[100]_{\mathrm{YBCO}} /[100]_{\mathrm{MgO}}$ in the sputtered regions. To be concise, hereafter we refer to the former as a " $45^{\circ}$ orientation" and the latter as a " $0^{\circ}$ orientation", using the misorientation angle between YBCO [100] and MgO [100] to name the orientation relation. Although the SIEM method of making GBJs is simple the factors which affect the YBCO growth are quite complex. Many processes occur during low energy ion bombardment of the surface, including surface morphology change, implantation of the ions and modification of surface chemistry and structure. In order to reproducibly control the growth orientation and further improve the GBJ quality it is essential to know what factors are the most important ones and ultimately what is the mechanism responsible for the orientation change.

\section{Experimental}

The substrates used in this work were epitaxially polished $\mathrm{MgO}(001)$ chips. The ion source used for the sputtering treatment was a low voltage $3 \mathrm{~cm}$ Kaufman-type ion gun with a hot $W$ filament, made by IonTech, Inc. The base vacuum of the sputtering chamber was better than $1 \times 10^{-6}$ torr. Typical ion sputtering condition was: $500 \mathrm{eV}$ ion beam at $90^{\circ}$ incidence for 4 minutes with a current density of $\sim 1 \mathrm{~mA} / \mathrm{cm}^{2}$. Different ion energies, incident angles and milling times were also used to study the effects of these parameters on the resultant epitaxy. A similar ion source using no tungsten filament was also used to sputter samples to isolate the effect of the W filament. When making $45^{\circ}$ tilt boundaries, the substrates were patterned with a standard photolithography procedure to define the regions to be sputtered and the precise position of the boundaries. The POMBE method and the set-up for YBCO film growth have been reported elsewhere[4]. The organo-metallic precursors used were Y(dmp $)_{3}, \mathrm{Ba}(\mathrm{hfa})_{2}$.(tet) and $\mathrm{Cu}(\mathrm{dpm})_{2}$. The precursors were pulsed onto the substrates via a set of computer controlled 
valves, which allow very fine tuning of the pulse sequence and length and hence the type and amount of the precursors delivered to the substrates. Usually, the precursors are pulsed in the sequence of $\mathrm{Ba}-(\mathrm{Cu}-\mathrm{Y}-\mathrm{Cu}-\mathrm{Ba}-\mathrm{Cu}-\mathrm{Ba}-)_{\mathrm{n}}$, where $n$ is the repeat number of the sequence in the parenthesis. The reason for the initial Ba pulse is to form a "seed layer" on the sputtered region. Only a certain amount of the initial Ba deposition can create the desired orientation relation and form $45^{\circ}$ boundaries. The details have been reported[5] previously and will be further discussed in the next sections. In the process of the film growth the substrate was heated to a temperature between $670^{\circ} \mathrm{C}$ to $685^{\circ} \mathrm{C}$ and treated with an oxygen plasma.

The film orientations were determined by $\mathrm{x}$-ray, electron backscatter channeling pattern (BSCP) in SEM and selected area electron diffraction in TEM. The morphology change of the substrate surface is examined by AFM and XTEM. The latter, alone with the high spatial resolution EDX, is also used to examine the structural and compositional change at the YBCO/MgO interfaces and the $45^{\circ}$ tilt boundaries. The microscopes used for TEM include a JEOL-4000EX, a Hitachi H-9000 and a Hitachi HF-2000 field emission analytical microscope. RBS was used to quantitatively study the surface chemistry of the sputtered substrates and the initial stage of the film growth. The RBS experiment is performed at the $2 \mathrm{MeV}$ Tandem National Electrostatics ion accelerator facility at Argonne National Laboratory. A $\mathrm{He}^{+}$beam of $1.6 \mathrm{MeV}$ is used for the analysis. The TRIM program is used to simulate the path of the Ar and $\mathrm{W}$ ions and their spatial distribution in the MgO substrate.

\section{Results and discussion}

Under favorable sputtering and growth conditions good single-crystal-like YBCO thin films with their c-axis aligned with the substrate surface normal and a- , b-axes locked in two perpendicular directions can be readily obtained. Fig.1a,b are x-ray diffraction intensity spectra of the YBCO films grown on sputtered and non-sputtered substrates, respectively. As indicated by the spectra, both films have good crystallinity. The in-plane orientation of the films are $[100]_{\mathrm{YBCO}} / /[100]_{\mathrm{MgO}}\left(0^{\circ}\right.$ orientation $)$ for the sputtered substrate and $[110]_{\mathrm{YBCO}} / /[100]_{\mathrm{MgO}}\left(45^{\circ}\right.$ orientation) for the non-sputtered substrate, indicating the occurrence of the sputter-induced epitaxy modification (SIEM). Under un-favorable synthesis conditions mixed orientations may occur. In those situations both sets of peaks will appear in the spectra. The ratio of the area of one orientation relative to the other can be determined by the relative height of the $\mathrm{x}$-ray peaks. Using this ratio one can tell whether SIEM occurs in a specific experimental condition and to what extent the orientation relation is changed. The orientation change can also be studied by BSCP which allows the orientation determination in areas as small as a few microns.

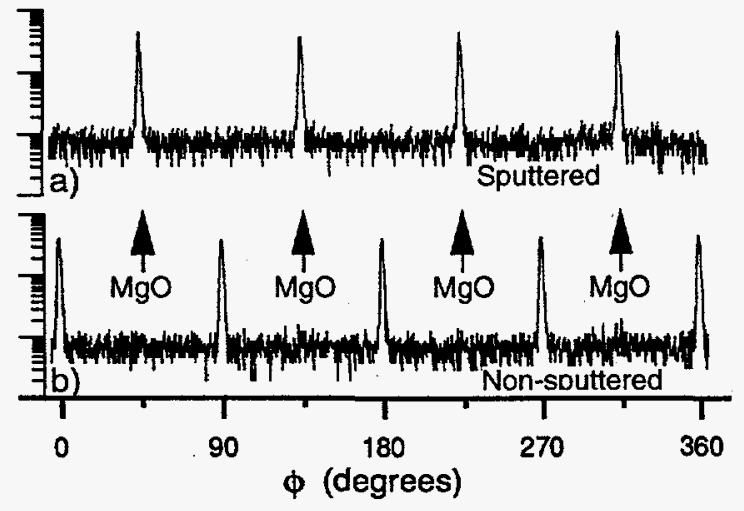

Fig.1 $\phi$-scan X-ray diffraction intensity spectra of YBCO films grown on a)sputtered and b)nonsputtered $\mathrm{MgO}(001)$ substrates.
The effect of the three most important sputtering parameters, ion beam energy, ion incident angle and sputtering time, on the occurrence of SIEM are explored in a film growth experiment. The results are compiled in table 1. From table 1 one can see that SIEM does not occur at low ion energy, shallow incident angle and short sputtering time. In each of the cases a threshold value appears to exist below which SIEM does not occur. The thresholds of time and energy are easy to understand, because below the thresholds the impact of the ion beam on the substrate is small. The dependence on the ion beam incident angle may shed some light onto the mechanism involved. It is well known that the ion sputtering at shallower incident angle (e.g. $30-40^{\circ}$ ) results in faster removal of materials from the surface and larger surface damage than at perpendicular incidence. Therefore, the observed trend seem to imply that the surface damage or surface morphology change caused by ion sputtering is not the major factor causing SIEM. Should surface damage, such as formation of steps, ledges and facets, leads to SIEM, SIEM would occur on the surface sputtered at low angles more easily. 
Ion energy (eV) Incident Angle( $\left(^{\circ}\right)$ Time (min) SIE (Yes/No)

\begin{tabular}{llll}
\hline \hline 50 & & & $N$ \\
100 & & 3 & $Y$ \\
200 & 90 & & $Y$ \\
300 & & $Y$ \\
500 & & $Y$ \\
\hline & 10 & & $N$ \\
& 30 & 3 & $N$ \\
300 & 45 & & $Y$ \\
& 60 & & $Y$ \\
& 75 & 1 & $Y$ \\
& 90 & 2 & $N$ \\
& & 3 & $Y$ \\
300 & & 4 & $Y$ \\
& 90 & 6 & $Y$ \\
& & & $Y$ \\
\hline
\end{tabular}

Table 1 Occurrence of sputter-induced epitaxy of YBCO on MgO (001) substrate at different sputtering conditions
To study possible implanted species, the composition change of the substrate surface layer during the ion sputtering was examined by RBS. Fig. 2 shows a typical RBS spectrum of the $\mathrm{MgO}$ substrate sputtered by the Ar ion gun with W filament. In addition to the high $\mathrm{Mg}$ and $\mathrm{O}$ edges there are two small extra peaks in the spectrum corresponding to $\mathrm{Ar}$ and $\mathrm{W}$, which are introduced into the substrate during ion-sputtering. Ar comes from the beam itself, whereas the source of the $\mathrm{W}$ is the hot filament of the gun. During sputtering W atoms are emitted by the filament, ionized and accelerated along with the Ar ions. This is consistent with the large $W$ filament consumption

during sputtering. TRIM code Monte Carlo simulations suggest that the Ar and W atoms are implanted into the top $3 \mathrm{~nm}$ layer of the $\mathrm{MgO}$ substrate and the $\mathrm{W}$ implantation is slightly deeper than Ar. Assuming these spatial distributions, the RBS spectra were simulated and the $\mathrm{Ar}$ and $\mathrm{W}$ concentrations in the $\mathrm{MgO}$ surface layer were determined by matching the simulated spectrum to the experimental spectrum. At the typical sputtering condition described in

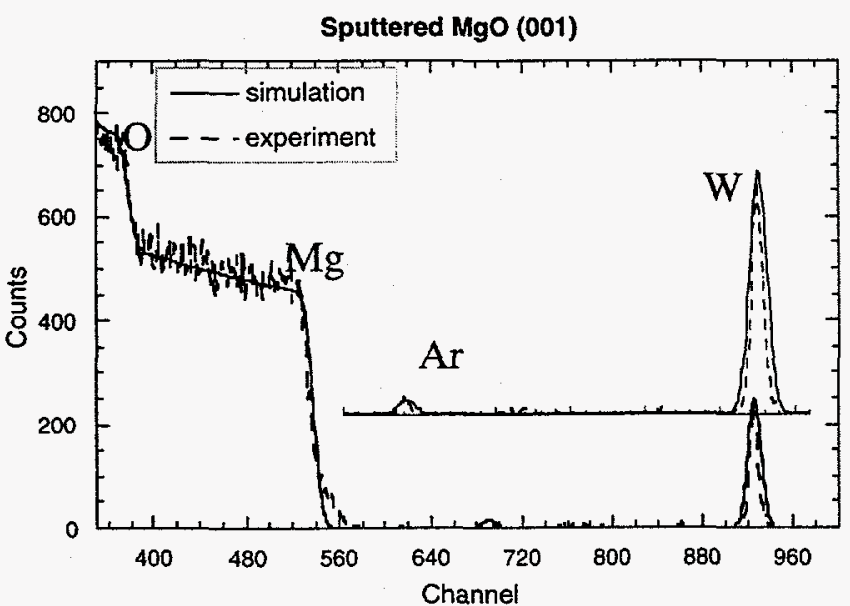

Fig.2 RBS spectrum of sputtered $\mathrm{MgO}(001)$ substrate showing $\mathrm{Ar}$ and $\mathrm{W}$ peaks. section 2 the total amount of Ar and W in the surface layer are about 1.1 and 0.8 monolayers, respectively. The substrates are usually treated for 30 minutes with an oxygen plasma at $\sim 700^{\circ} \mathrm{C}$ before starting the YBCO growth. RBS results show that most of the Ar and W atoms survive after this treatment. This confirms that the $\mathrm{Ar}$ and $\mathrm{W}$ atoms are indeed implanted into the substrate.

The Ar and $\mathrm{W}$ concentration in the substrate surface layer increases with sputtering time (fig.3a). However, the $\mathrm{Ar}$ concentration saturates in less than one minute of sputtering. This suggests that $\mathrm{Ar}$ implantation may not be important to SIEM because according to table 1 no

SIEM has occurred yet at such a low exposure to the ion beam. The $\mathrm{W}$ implantation, on the other hand, may have an important effect on the change of the film orientation. This can be seen from fig. $3 \mathrm{~b}$ which shows that the concentration of $\mathrm{W}$ and the fraction of $0^{\circ}$ oriented phase both increase with the increasing incident angle, suggesting a connection between the $W$ implantation and the occurrence of SIEM. To further explore this possible connection, YBCO films are grown on substrates sputtered by an Ar ion gun without W filament, with the other conditions being the same. In the resultant films the fraction of the $0^{\circ}$ phase is only slightly larger than that of non-sputtered case, indicating that W-free Ar ion sputtering has little effect on the occurrence of SIEM and hence W implantation is essential to SIEM.

The YBCO film growth conditions also affect its orientation on the $\mathrm{MgO}$ substrate. Among others, the length of the initial $\mathrm{Ba}$ pulse is found to be an important factor. The result is illustrated in fig. 4 , which plots the fraction of $0^{\circ}$ oriented areas versus the initial $\mathrm{Ba}$ pulse time. The three curves show the results for the $\mathrm{MgO}$ (100) substrates non-sputtered, sputtered by $\mathrm{Ar}$ ion gun with and without a W filament, respectively. In all three situations the YBCO films 


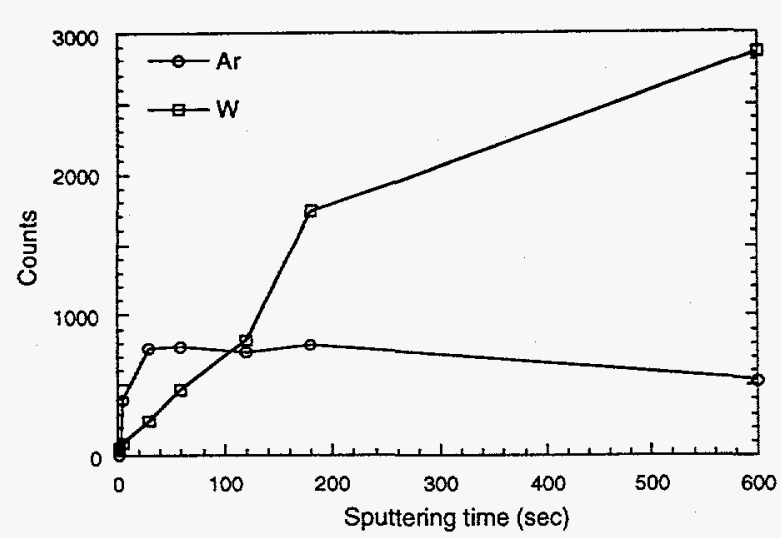

Fig.3a Change of implanted Ar and W concentration with sputtering time. Ar saturates in less than 0.5 minutes.

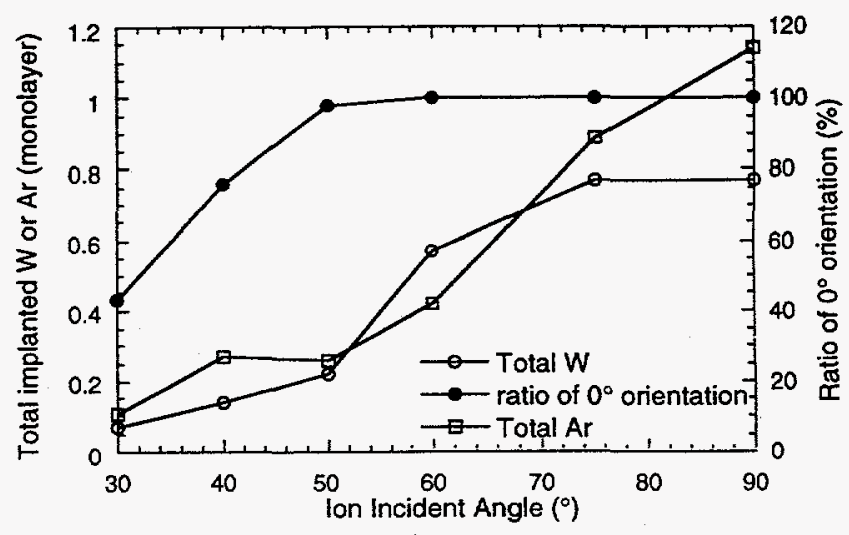

Fig.3b Amount of implanted $\mathrm{W}$ and the $0^{\circ}$ oriented phase both increase with sputtering angle.

grow entirely in the $0^{\circ}$ orientation when the initial $\mathrm{Ba}$ pulse is longer than 9 seconds. This means that if we deposit a significant amount of Ba onto the $\mathrm{MgO}$ surface before the YBCO growth we always get $0^{\circ}$ oriented YBCO film no matter what treatment was done to the substrate. This phenomenon can be called "Ba induced $0^{\circ}$ growth". When the initial Ba pulse is short, however, the film orientation can be different. For each of the three cases there is a critical $\mathrm{Ba}$ pulse length below which the resultant YBCO film switches to the $45^{\circ}$ oriented film.

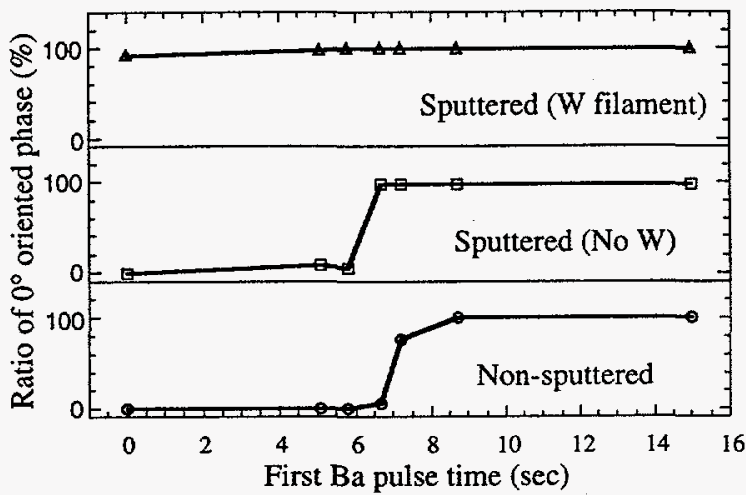

Fig.4 Fraction of $0^{\circ}$ oriented phase changes with the first $\mathrm{Ba}$ pulse time and the treatment to the substrate.
The switching of the orientation is quite sharp. Ion sputtering changes the value of the critical Ba pulse length. A W-free sputtering only changes this value slightly, but sputtering by an ion gun with a W filament reduces this value from about 7.1 second to almost 0 , forming a window in which simultaneous growth of $0^{\circ}$ and $45^{\circ}$ oriented phases on sputtered and non-sputtered substrate respectively becomes possible.

The "Ba induced $0^{\circ}$ growth" has been explained by Buchholz et al[4] using Cotter's results on the initial growth behavior of $\mathrm{BaO}$ on the $\mathrm{MgO}(001)$ surface. In their

experiment Cotter et al [6] found that at a Ba coverage less than a critical coverage $(\sim 2$ monolayer) the $\mathrm{Ba}$ atoms are substitutionally incorporated into the $\mathrm{MgO}$ lattice but if the coverage is greater than the critical value an overlayer $\mathrm{BaO}$ was formed. The c-axis of this overlayer is aligned with that of $\mathrm{MgO}$ and the in-plane orientation relation is $[110]_{\mathrm{BaO}} / /[100]_{\mathrm{MgO}}$. This observation was justified by calculating the heat of segregation[7] for the two situations. Buchholz et al. suggested that the Cotter's critical $\mathrm{Ba}$ coverage for the $\mathrm{BaO}$ overlayer to form corresponds to the critical initial Ba pulse length in the POMBE growth. Above the critical $\mathrm{Ba}$ coverage a $\mathrm{BaO}$ overlayer is formed. The subsequent YBCO films grow on the $\mathrm{BaO}$ overlayer with the orientation relation $[100]_{\mathrm{YBCO}} / /[110]_{\mathrm{BaO}}$, giving the overall $0^{\circ}$ orientation relation between YBCO film and $\mathrm{MgO}$ substrate.

The above suggested growth mode could lead to a possible mechanism of SIEM based on the following speculation. If the sputtering increases the decomposition rate of the $\mathrm{Ba}$ precursor by modifying the surface morphology and/or chemistry, more Ba would be produced on the sputtered areas and a "Ba-induced $0^{\circ}$ growth" could occur on those areas. This mechanism, however, was shown not to be correct by a critical experiment in which $\mathrm{Ba}$ is deposited onto a sputtered and a non-sputtered substrates at the same time. In this experiment RBS results showed no significant difference in the surface Ba concentration between the sputtered and non-sputtered substrates. Hence, the mechanism of SIEM is not "Ba induced $0^{\circ}$ growth". 


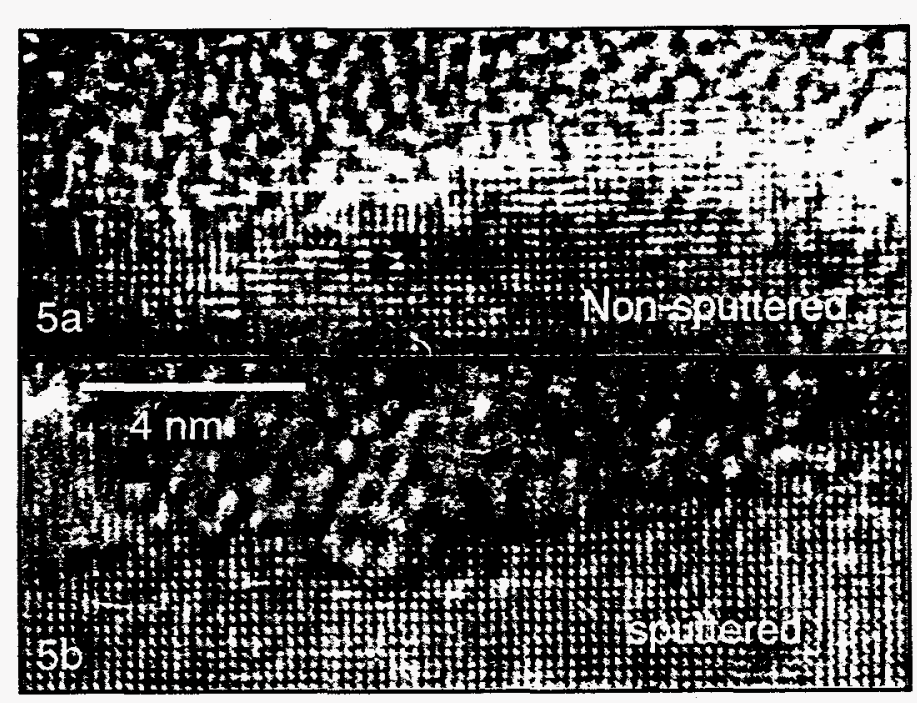

Fig.5 Surface profile images of a) non-sputtered and

b) sputtered $\mathrm{MgO}(001)$ substrates showing no obvious difference in surface morphology. morphology, such as steps in $<100\rangle$ directions, which would favor $0^{\circ}$ growth. The observation on the surface morphology by AFM and XTEM, however, seems negative to this mechanism. AFM images show that the surface of the as-sputtered substrate is rougher than that of non-sputtered substrate, but after an hour of annealing in an oxygen plasma the morphology of the two surfaces become similar, except that height fluctuations on the sputtered substrates are slightly larger than those on the non-sputtered substrates. The XTEM images (fig.5) exhibit a similar tendency: height fluctuations on the sputtered surface is larger, but otherwise there are no striking difference between sputtered and non-sputtered substrates regarding atomic-scale features in the surface profile. These observations reinforce the conclusion reached at the

beginning of this section, that the morphology change caused by ion sputtering is not an important factor in SIEM.

In order to characterize the structural changes due to ion sputtering, YBCO/MgO interfaces on non-sputtered(fig.6a) and sputtered(fig.6b) substrates are examined by XTEM. The images

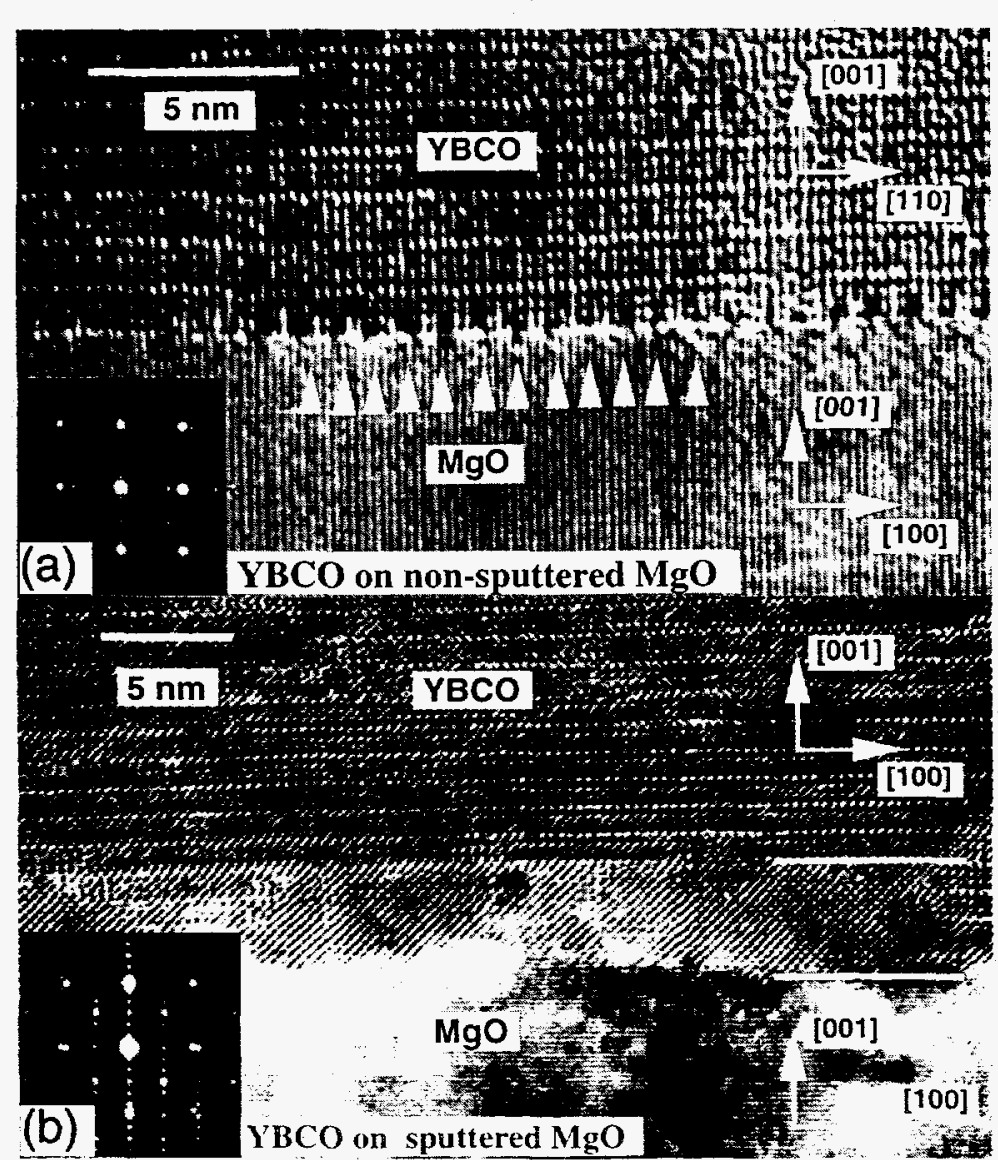

Fig. 6 YBCO/MgO interfaces on the a)non-sputtered and $b$ ? sputtered substrates. The latter has an intermediate layer. show remarkable differences between the two interfaces. The non-sputtered one is sharp and clean. The YBCO films grow directly on the MgO surface. There are no reaction products and intermediate layer at the interface. The periodical features at the interface indicated by arrows suggest the formation of interfacial dislocations which match every four $\mathrm{MgO}[200]$ planes with three YBCO [220] planes. The sputtered interface, on the other hand, is not sharp. It is characterized by an intermediate layer 3-6 nm thick. This layer displays different lattice fringes compared to other parts of the sample, indicating that it has a structure different from YBCO and $\mathrm{MgO}$. The elemental distribution at the sputtered and non-sputtered interfaces are also different. Shown in fig. 7 are elemental profiles obtained by EDX line scan across the interfaces. In the non-sputtered sample the interface looks sharp. Although there is some diffusion of $\mathrm{Mg}$ into YBCO there is no diffusion of $\mathrm{Y}, \mathrm{Ba}$ and $\mathrm{Cu}$ into $\mathrm{MgO}$. At the 
sputtered interface, however, a considerable amount of $\mathrm{Ba}$ diffuses into the $\mathrm{MgO}$ region and forms a Ba-Mg mixed region 4-6 nm thick. Also, a small amount of $\mathrm{W}$ is detected in the interface region, consistent with the RBS result mentioned above.

From the sum of the experimental results the following picture emerges regarding the process occurring on the MgO surfaces in the early stage of YBCO film growth. On the nonsputtered $\mathrm{MgO}$ surface the film grows "normally" with no intermediate layer formed. Because

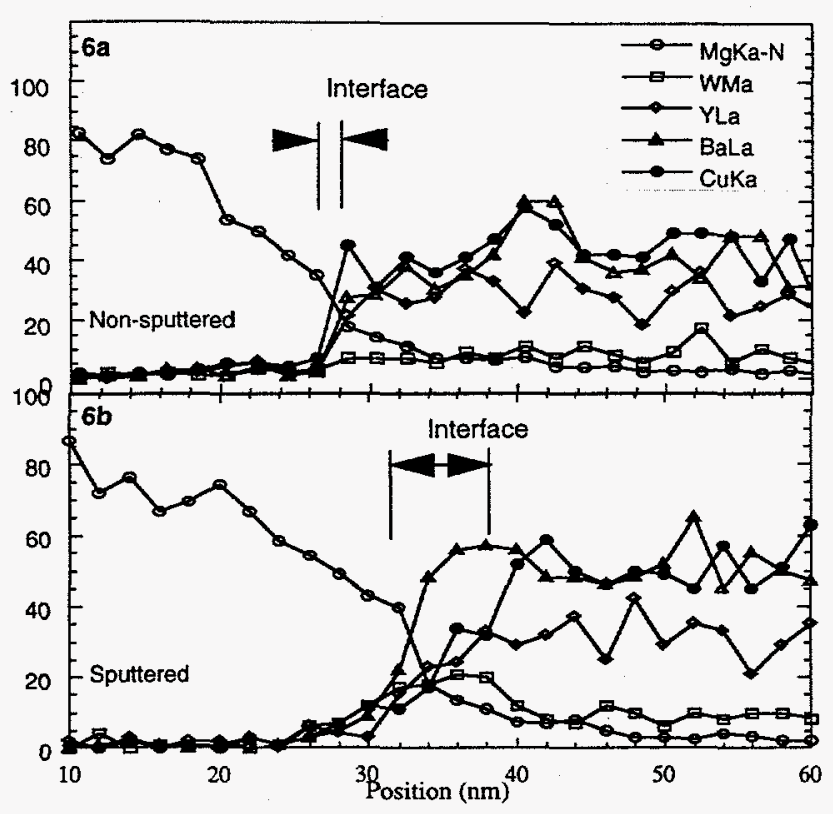

Fig.7 Elemental profiles across the YBCO/MgO interfaces in the films grown on a)non-sputtered and b)sputtered substrates. The non-sputtered interface is sharp. The sputtered interface has a Ba-rich intermediate layer. the $45^{\circ}$ orientation has a smaller lattice mismatch with the substrate the film grows exclusively in this orientation. On the sputtered surface, however, the W implantation, and to some extent the Ar sputtering, introduce some kind of structural and chemical changes in the surface layer of the substrate and lead to a strong $\mathrm{Ba} / \mathrm{MgO}$ interdiffusion. This interdiffusion forms a layer of $\mathrm{Mg}_{\mathrm{x}} \mathrm{Ba}_{1-\mathrm{x}} \mathrm{O}$ on the substrate surface which functions as a buffer layer for the subsequent film growth. The formation of the buffer layer avoids the large lattice mismatch between $\mathrm{MgO}$ and $0^{\circ}$ oriented YBCO film and makes the $0^{\circ}$ oriented growth energetically favorable.

\section{Conclusions}

The mechanism of the sputter-induced orientation change in YBCO growth on $\mathrm{MgO}$ (001) substrate has been investigated. Several effects are considered. The surface morphology change and Ar implantation have been excluded as major factors by the

experiments regarding the occurrence of SIEM at different sputtering conditions. The structural and chemical changes introduced by the $\mathrm{W}$ implantation during the ion sputtering are found to be a major factor. Because of these changes, $\mathrm{Ba}$ atoms diffuse into the MgO substrate and form a $\mathrm{Mg}_{\mathrm{x}} \mathrm{Ba}_{1-\mathrm{x}} \mathrm{O}$ layer on the sputtered surface. No such layer is formed on the non-sputtered surface. The $\mathrm{Mg}_{\mathrm{x}} \mathrm{Ba}_{1-\mathrm{x}} \mathrm{O}$ layer formed on the sputtered substrate functions like a seed layer in the bi-epitaxy method which introduces an extra orientation rotation to the subsequent YBCO film and makes the final orientation on the sputtered substrate surface different from that on the non-sputtered surface.

\section{Acknowledgment}

The authors want to thank V Dravid, W.A.Chiou, Y.Y.Wang for help in the microanalysis, to R.Csencsits, H.Zhang, and W.Sinkoer for their help in the HREM experiments and to L.J.Thompson for help with TRIM and RBS simulation. This work is supported by the National Science Foundation, under contract \#DMR 91-20000 (YH, BVV) and the U. S. Department of Energy under contract \#W-31-109-ENG-38 (KLM).

\section{References}

[1] B.V.Vuchic et al, J. Appl. Phys. 77 (1995) 2591.

[2] D.B.Buchholz et al, Appl. Phys. Lett. 68 (1996)3037.

[3] N.G.Chew et al, APL 60 (1992) 1516.

[4] D.B.Buchholz et al, Mater.Chem.Phy. 36 (1994) 377.

[5] D.B.Buchholz et al, submitted to J. Alloys and Compounds.

[6] M.Cotter et al, Surf. Sci., 197 (1988) 208.

[7] M.Cotter et al, Surf. Sci., 208 (1989) 267. 